\title{
Turbulent transport and turbulence in radiative I mode plasmas in TEXTOR-94
}

\author{
J.A. Boedo ${ }^{\mathrm{a}}$, J. Ongena ${ }^{\mathrm{b}}$, R. Sydora ${ }^{\mathrm{c}}$, D.S. Gray ${ }^{\mathrm{a}}$, S. Jachmich ${ }^{\mathrm{b}}$, \\ R.W. Conn ${ }^{\mathrm{a}}$, A. Messiaen ${ }^{\mathrm{b}}$, TEXTOR Team ${ }^{\mathrm{d}}$ \\ ${ }^{a}$ Fusion Energy Research Program, \\ Department of Applied Mechanics and Engineering Sciences, \\ University of California, San Diego, \\ Jacobs School of Engineering, \\ La Jolla, California, United States of America \\ b Laboratoire de Physique des Plasmas-Laboratorium voor Plasmafysica, \\ École Royale Militaire-Koninklijke Militaire School, \\ Association 'Euratom-Belgian State', \\ Brussels, Belgium \\ ${ }^{c}$ Department of Physics, \\ University of Alberta, \\ Edmonton, Alberta, Canada \\ d Max-Planck-Institut für Plasmaphysik, Forschungszentrum Jülich, \\ Jülich, Germany
}

\begin{abstract}
First measurements of turbulence levels and turbulence induced transport in the outer edge of the plasma of TEXTOR-94 during radiative improved mode discharges show a reduction by a factor of $4-7$ of the radial particle turbulent transport. The quenching is most evident on the normalized potential fluctuations and is strongest above $100 \mathrm{kHz}$. Non-linear gyrokinetic particle-incell simulations of these discharges show impurity induced suppression of the electrostatic fluctuations associated with the ion temperature gradient driven mode over most of the cross-section, including the edge. Such a mechanism is proposed as the explanation for the improved confinement and turbulence reduction. The reduction in the edge turbulent transport levels is consistent with increased particle confinement time and the reduction of the SOL thickness. Particle and energy fluxes to the limiter are reduced by an order of magnitude. A concomitant increase of the measured energy and particle confinement times $\tau_{E}$ and $\tau_{p}$ versus radiated fraction suggests a common underlying suppression mechanism.
\end{abstract}

\section{Introduction}

Tokamak plasmas can undergo transitions from a low energy confinement state (L mode) to a higher energy confinement state [1] (H mode) spontaneously. The transition is accompanied by a negative radial electric field inside the LCFS and is characterized by a steepening of the edge profiles or the formation of a transport barrier. Simultaneously, there is a fast reduction of the $\mathrm{H}_{\alpha}$ signal, corresponding to increased particle confinement. Similar behaviour to the spontaneous $\mathrm{L}-\mathrm{H}$ transition was obtained in the tokamak CCT [2] by applying an external radial electric field to the edge plasma by biasing or 'polarizing' an electrode. As such, the CCT experiments suggested that the radial electric field and induced poloidal rotation play a crucial role in the $\mathrm{L}-\mathrm{H}$ transition. The stabilization of turbulence by $\boldsymbol{E} \times \boldsymbol{B}$ velocity shear is a general mechanism that has been proposed to explain stabilization of edge turbulence [3] in tokamaks and is supported by early work at the TEXT tokamak [4].

A new confinement regime called the radiative improved (RI) mode $[5,6]$ has been observed on TEXTOR-94. This regime is produced by injecting trace amounts of a gas such as argon or neon into the discharge $[7,8]$. This results in a strongly radiating belt, located in the plasma edge, where the various atomic processes produce a peak in the radiation rate for the given impurity gas. This radiating plasma regime allows a homogeneous dissipation of the discharge power over the inner wall of the tokamak, thereby reducing the power load on plasma facing components (PFCs). 


\section{J.A. Boedo et al.}

In addition to the reduced power load to the PFCS, the RI mode is characterized by high confinement (similar in quality to either ELM-free or ELMy $\mathrm{H}$ mode operation). The energy confinement time increases linearly with the plasma density (up to or above the Greenwald density limit), thereby recovering the so-called neo-Alcator scaling [9]. In addition, RI mode discharges exhibit high beta (up to the previously observed beta limits of TEXTOR, i.e. $\beta_{n}=2$ or $\beta_{p}=1.5$ ) and negligible deleterious effect of the seeded impurity on the fusion reactivity, thereby offering the possibility of operation at low edge $q$ under quasi-stationary conditions [10]. Apart from extending the performance and the operating range of the RI mode, the main effort of the TEXTOR-94 programme is devoted to the investigation of how these operational conditions are linked to the physical mechanism(s) leading to the confinement improvement.

The velocity shear stabilization mechanism is now proposed to be responsible for a series of improved confinement regimes in tokamaks including the $\mathrm{H}$ mode [11] and $\mathrm{V}-\mathrm{H}$ mode [12]. Recent experiments in TFTR have related the levels of local $\boldsymbol{E} \times \boldsymbol{B}$ shearing rates [13] to core transport barriers in reverse magnetic shear plasmas [14]. Work at DIII-D [15] and PBX-M [16] showed that the turbulence is reduced by the triggering of the spontaneous $\mathrm{H}$ mode as the result of concomitant changes in the radial electric field and the profile gradients. Polarization experiments in the TEXTOR tokamak $[17,18]$ have confirmed earlier CCT and TEXT results and significantly expanded the understanding of the role of the radial electric field and its bifurcation [19]. More recent experiments in TEXTOR-94 have shown the formation of a transport barrier in the plasma edge [20] as determined by the changes in the density profiles during polarization [21] and have correlated those changes to the gradients of the radial electric field. Clear correlation between externally applied electric fields and a reduction in turbulence levels in TEXTOR was first shown by our early work [22] and completed recently [23].

Interestingly, the concept of a fusion reactor with a radiating mantle has been of long standing interest. More recently, the experimental programme on reversed field pinches (RFPs) associated with the ZT-40M experiments [24] and modelling studies performed in conjunction with the TITAN RFP reactor study $[25,26]$ demonstrated stable plasma operation for plasmas radiating up to $85 \%$ of the input power [27]. However, the experiments in TEXTOR were the first to rediscover the earlier results of ISXB $[28,29]$ and demonstrate this type of operation in a tokamak. Since then, this regime has been found in a variety of diverted and limited tokamaks such as ASDEX-Upgrade, DIII-D and Tore Supra [30]. It is important to the fusion community to improve our understanding of this new tokamak confinement regime because it opens the possibility of operating a tokamak fusion reactor using a radiative mantle, dissipating the energy uniformly over the walls. A limiter configured fusion reactor may even become a possibility. The homogeneous dissipation will reduce the heat flux to the divertor components - one of the critical issues in the design of ITER. A fusion reactor operating in this regime could simplify the design, and reduce the cost, of fusion reactors.

Another mechanism in consideration for explaining turbulence reduction and one that does not involve $\boldsymbol{E} \times \boldsymbol{B}$ velocity shearing is the direct stabilization of ion temperature gradient-driven (ITG) turbulence and the hybrid ITG trapped electron drift instabilities by the radiating impurities. The ITG instability is driven by the interaction between the toroidal curvature, non-uniform toroidal magnetic field and the radial ion thermal gradient in the plasma. Vertical ion drifts due to the curvature and $\nabla B$ drift are functions of energy, therefore, different drift rates due to the ion energy distribution lead to density and electrostatic potential perturbations. The resultant $\boldsymbol{E} \times \boldsymbol{B}$ convection pattern produces an interchange of hot and cold plasma regions so that an initial temperature perturbation will grow if hotter plasma is convected into the enhanced temperature region of the initial perturbation. The linear stabilizing effect of impurities, especially those with highly charged states, on ITG modes has been considered previously [31-35]. This effect can be most easily seen from the fluid limit growth rate of the ITG mode including impurities and neglecting the parallel ion dynamics. For the small impurity fraction limit, $Z n_{Z} / n_{e}<1$, where $Z$ is the impurity charge number and $n_{Z}$ the number density of the impurity, along with $T_{i}=T_{Z}, L_{n}=L_{n Z}$ and $L_{T}=L_{T Z}$, we obtain the interchange type growth rate

$\gamma_{I T G} \approx \sqrt{\left[1-(Z-1) n_{Z} / n_{e}\right] \omega_{*_{p i}} \bar{\omega}_{D i}}$

where

$1 / L_{n}=\frac{-1}{n} \frac{d n}{d r}, \quad \omega_{*_{p i}}=\frac{k_{\theta} c}{e B n_{i}} \frac{d p_{i}}{d r}$ 
Article: Turbulent transport and turbulence in RI mode

is the ion pressure gradient drift frequency, $k_{\theta}$ is the poloidal wavenumber

$\bar{\omega}_{D i}=\frac{L_{n}}{R} \omega_{*_{p i}} \frac{\left\langle v_{\perp}^{2}+v_{\|}^{2} / 2\right\rangle}{v_{t h i}^{2}}$

is the energy averaged ion toroidal drift frequency, $\rho_{i}=c \sqrt{m_{i} T_{i}} / e B$ is the ion gyroradius and $v_{t h i}=$ $\sqrt{T_{i} / m_{i}}$. A Boltzmann response for the electrons can be used if one assumes that the phase velocity of the perturbation satisfies $v_{t h e}>\omega / k_{\|}>v_{t h i}$. In the small impurity fraction limit, the real frequency of the mode is $\omega_{\text {real }} \sim \omega_{D i}>\omega_{D Z}$. From Eq. (1), it can be seen that impurities reduce the driving term of the ITG mode by:

(a) Diluting the main ion species,

(b) Modifying the pressure or density gradient (and thereby $\omega_{*_{p i}}$ or the scale length $L_{n}$ ).

The stabilizing trend persists in a more complete kinetic calculation of the growth rate which includes the parallel ion dynamics, however the initially stabilizing effect of the impurities becomes destabilizing when $Z n_{Z} / n_{e}$ increases. Maximum stabilization occurs when the amounts of the two species (deuterium, neon) are equal in terms of the charge density fraction. Linear theory analysis indicates that the ITG and ITG trapped electron modes are unstable in the core and edge regions of TEXTOR discharges, therefore, the presence of impurities such as neon, which contain high charge states, is strongly stabilizing for such instabilities.

New observations on the behaviour of the turbulence levels and the radial turbulent particle flux in the outer plasma edge, as measured with a fast scanning probe, are presented in this article. A reduction in the RMS levels of density and poloidal electric field result in diminished radial particle flux. Non-linear simulation results are presented and it is proposed that the turbulence reduction is a direct consequence of the neon seeding causing a reduction in the growth rate and saturation levels of low frequency microturbulence arising from ITG instabilities. The reduced radial particle flux is correlated with the measured increase in particle and energy confinement. The signature of velocity shear stabilization is not present on the turbulence data during RI mode, in either the space or frequency domain. It is important to mention that the RI mode confinement seems to be a fairly continuous function of radiated fraction and it is triggered at $\gamma \sim 0.6$.

\section{Experiment}

TEXTOR-94 is a long pulse medium size, circular cross-section tokamak (major radius $R=1.75 \mathrm{~m}$, minor radius $a=0.46 \mathrm{~m}$, pulse lengths of about $10 \mathrm{~s}$ ) equipped with the pumped toroidal belt limiter ALT-II [36]. The experiments described below are conducted in quasi-stationary RI mode discharges on TEXTOR-94. The discharges have a radiated power fraction $\gamma=P_{r a d} / P_{t o t}$ (where $P_{t o t}$ is the total input power and $P_{\text {rad }}$ is the radiated power) between 0.3 and 0.95 . The other discharge parameters are: plasma current $I_{p}=400 \mathrm{kA}$, toroidal magnetic field $B_{t}=2.25 \mathrm{~T}$ and line averaged central density, $\bar{n}_{e}$, between 4 and $6 \times 10^{13} \mathrm{~cm}^{-3}$. Additional heating of up to $2-3 \mathrm{MW}$ consists of co-injection $\left(\mathrm{D}^{+} \rightarrow\right.$ $\mathrm{D}^{0}$ injection at about $48 \mathrm{keV}$ ) combined with ICRF heating (at $\omega=2 \omega_{C D}$, i.e. at a frequency of $32 \mathrm{MHz}$, with $\pi$ phasing of the two antenna pairs, one with and one without a Faraday screen). The energy content of the plasma during the stationary phase has been feedback controlled by acting on the level of ICRF heating. The radiation level is kept constant by feedback control of the neon gas inlet valve using the intensity of a Ne VIII line [37]. The sink for neon particles is provided by the pump limiter ALT-II [38].

Profiles of electron temperature $T_{e}(r)$, density $n_{e}(r)$, saturation current $I_{\text {sat }}(r)$, poloidal electric field $E_{\tau}(r)$, floating potential $V_{f}(r)$ and fluctuations, were obtained using a fast reciprocating probe array [39] located in the low field edge of the plasma. The probe penetrates $2 \mathrm{~cm}$ inside the LCFS of TEXTOR$94(45.6 \mathrm{~cm}<r<50 \mathrm{~cm}, r$ is the minor radius) under these high power conditions. The data for the turbulent measurements are digitized at $1 \mathrm{MHz}$ with a 10 bit resolution digitizer and filtered by low pass, $500 \mathrm{kHz}$, anti-aliasing filters. We find that the power spectra of all the signals decays very quickly with frequency and is significant only up to $200 \mathrm{kHz}$. The turbulent particle flux $\tilde{\Gamma}_{r}$ is calculated in terms of the RMS values of the density $\left\langle\tilde{n}_{e}^{2}\right\rangle^{1 / 2}$, poloidal electric field $\left\langle\tilde{E}_{\theta}^{2}\right\rangle^{1 / 2}$ fluctuations and the local toroidal field $B_{\phi}$, which are measured by the probe array, as [40]

$$
\begin{aligned}
\tilde{\Gamma}_{r} & =\frac{\left\langle\tilde{E}_{\theta} \tilde{n}_{e}\right\rangle}{B_{\phi}}=\frac{\left\langle\tilde{n}_{e}^{2}\right\rangle^{1 / 2}\left\langle\tilde{E}_{\theta}^{2}\right\rangle^{1 / 2}}{B_{\phi}} \frac{\left\langle\tilde{E}_{\theta} \tilde{n}_{e}\right\rangle}{\left\langle\tilde{n}_{e}^{2}\right\rangle^{1 / 2}\left\langle\tilde{E}_{\theta}^{2}\right\rangle^{1 / 2}} \\
& =\frac{\left\langle\tilde{n}_{e}^{2}\right\rangle^{1 / 2}\left\langle\tilde{E}_{\theta}^{2}\right\rangle^{1 / 2}}{B_{\phi}} \gamma_{\tilde{E} \tilde{n}} \cos \alpha
\end{aligned}
$$

where the brackets denote ensemble averaging and the product of the coherence, $\gamma_{\tilde{E} \tilde{n}}$, and the cosine 


\section{J.A. Boedo et al.}
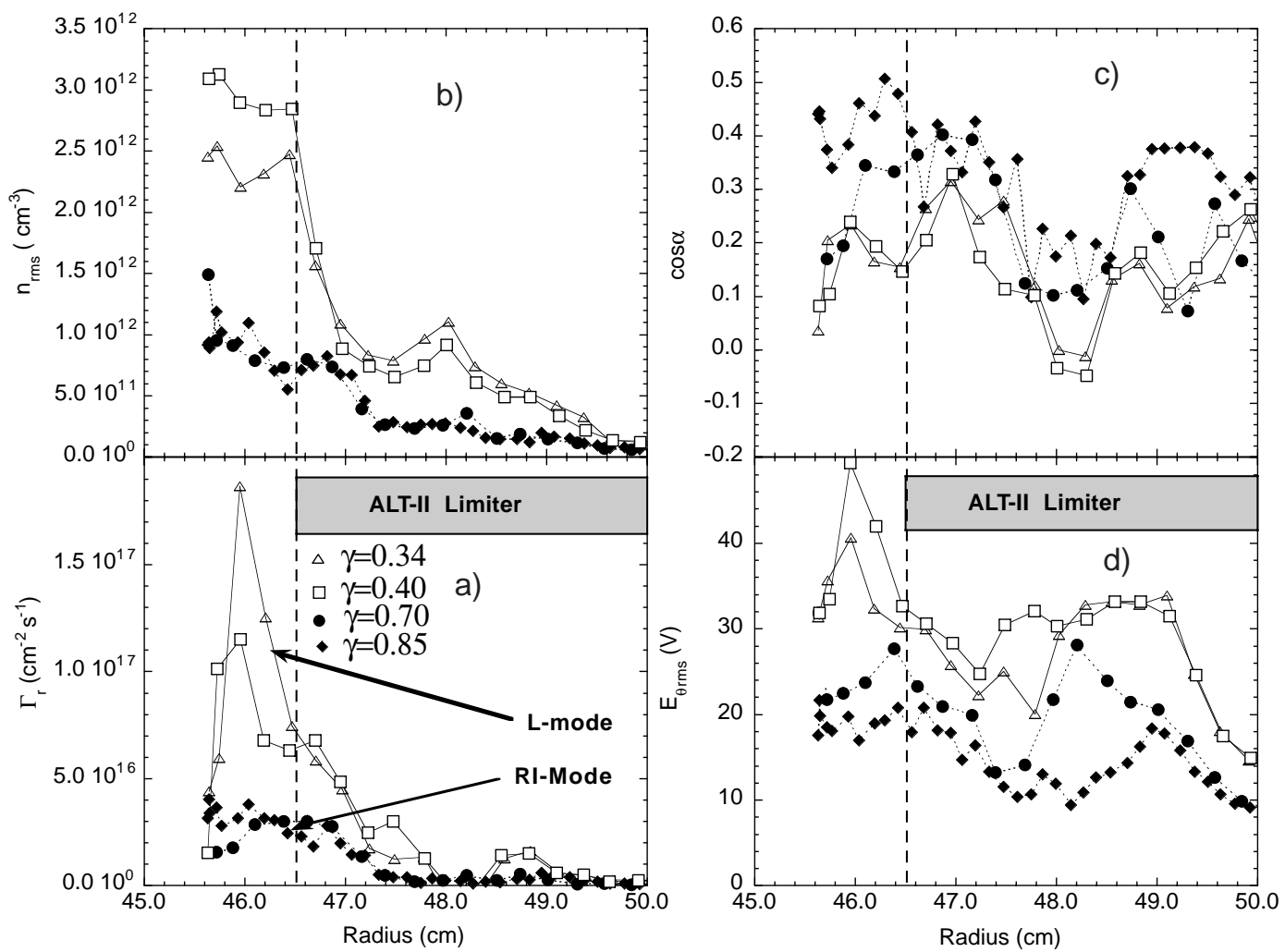

Figure 1. Radial profiles of (a) radial turbulent particle flux $\tilde{\Gamma}_{r}$, (b) density RMS level, (c) phase angle between density and poloidal field RMS levels, $\alpha$, and (d) poloidal electric field RMS level for L mode (open symbols) and RI mode (solid symbols). The limiter position is shown as a shaded rectangle.

of the phase angle between the density and poloidal electric field fluctuations, $\cos \alpha$, is defined as

$\gamma_{\tilde{E} \tilde{n}} \cos \alpha=\frac{\left\langle\tilde{E}_{\theta} \tilde{n}_{e}\right\rangle}{\left\langle\tilde{n}_{e}^{2}\right\rangle^{1 / 2}\left\langle\tilde{E}_{\theta}^{2}\right\rangle^{1 / 2}}$

The particle flux can be calculated alternatively in frequency space in terms of the density and potential fluctuation cross-power spectra $P_{n \phi}(\omega)$, the poloidal wavenumber $k_{\theta}(\omega)$, and the phase between the density and potential fluctuations, $\alpha_{n \phi}(\omega)$, as [41]

$\Gamma=\frac{2}{B_{\phi}} \int\left|P_{n \phi}(\omega)\right| k_{\theta}(\omega) \sin \alpha_{n \phi}(\omega) d \omega$.

The reciprocating probe is located at the outer midplane of the tokamak (TEXTOR's edge plasma is toroidally symmetric due to the ALT-II toroidal belt limiter). However, the measurements are similar to those taken with a second probe located at the top of TEXTOR and separated $90^{\circ}$ poloidally from the primary probe.

\section{Results and discussion}

\subsection{Turbulence and turbulent radial flux}

The results (at the LCFS) obtained for otherwise identical L mode and RI mode discharges are

(a) Absolute density $n_{r m s}$ and poloidal field $E_{\theta r m s}$, fluctuation levels are reduced by factors of $\sim 2$ 3 as seen in Figs 1(b) and 1(d). Note that the Boltzmann relation, which is usually not fulfilled in the SOL/edge, is recovered during RI mode conditions (Figs 2(a,b)).

(b) Radial turbulent particle transport $\tilde{\Gamma}_{r}$ (Fig. 1(a)) is reduced by a factor of about 4-7; mostly due to the lower $n_{r m s}$ and $E_{\theta r m s}$ levels with a small increase due to cross-phase effects (Fig. 1(c)).

(c) Normalized potential fluctuation levels $e \tilde{\phi} / k T_{e}$ decreased by a factor of 2 (Fig. 2(b)).

(d) Normalized density fluctuation levels remain unchanged (Fig. 2(a)). 


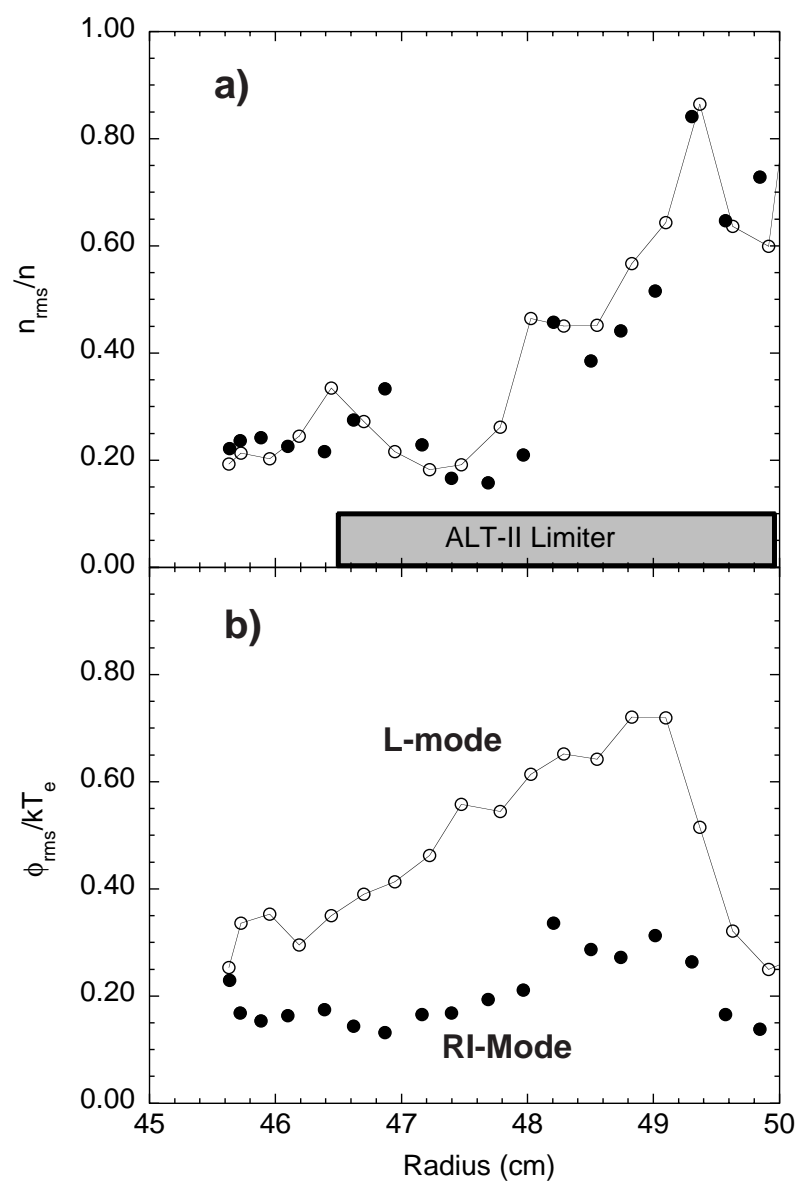

Figure 2. Radial profiles of (a) normalized density and (b) potential fluctuation levels as a function of radius for $\mathrm{L}$ mode and RI mode discharges. The normalized levels are more similar for RI mode conditions.

(e) The quenching of turbulent fluxes occurs at all frequencies (Fig. 3) but most strongly above $\sim 100 \mathrm{kHz}$ so the resulting particle transport is significant only at low frequencies.

(f) The edge radial electric field remains almost unchanged (the floating potential increases slightly inside the LCFS but the temperature decreases, so the plasma potential, $V_{p}=V_{f}+$ $3.5 k T_{e}$, is roughly constant, see Figs 5(a) and (b)), thus the turbulence reduction is not connected to an increase in the local $E_{r} \times B_{\phi}$ shear stabilization.

The turbulent quantities can also be examined in frequency space as per Eq. (3). The data samples between 45.5 and $46.0 \mathrm{~cm}$ (in the edge, not in the SOL) are used to assure good statistics, resulting in a spatial average over $\sim 0.5 \mathrm{~cm}$. Further averaging over frequency is performed in Figs $3(\mathrm{a}-\mathrm{d})$.
The main characteristic features remain constant for various radial positions. During the RI mode, the cross-power (Fig. 3(a)) and radial particle transport (Fig. 3(c)) are quenched at frequencies greater than 70-100 kHz. The cross-phase $\alpha_{n \phi}$ (Fig. 3(b)) does not vary significantly (except over a narrow range at very low frequencies) and $k_{\theta}$ (Fig. $3(\mathrm{~d})$ ) becomes more negative at lower frequencies. The coherence is about 0.6 across the spectrum, except at high frequencies, where the power drops. The data during RI mode lose significance above $250-300 \mathrm{kHz}$ due to the strong signal reduction.

To interpret the behaviour of turbulent fluctuations before and after neon injection, we consider a model in which ITG modes play a dominant role. There are several reasons to consider this source mechanism. First, linear theory calculations using TEXTOR experimental profiles show that ITG modes are unstable over most of the plasma volume and when radiating impurities are introduced the growth rate of these modes is reduced. Second, the reduction of the higher frequency portion of the turbulent fluxes, shown in Fig. 3, suggests that short wavelength ITG modes are suppressed since the frequency of the fluctuations is found to be proportional to the poloidal wavenumber. Finally, the modifications of the density and thermal profiles occurring after the RI mode transition are consistent with reduced levels of ITG turbulence. It should be emphasized that the model of ITG turbulence considered in this article should be considered as a first step in a more comprehensive turbulence model of the core-edge interaction. Further improvements to the model would include non-adiabatic electron effects arising from trapped electrons and electronion collisions, which have been found to increase the growth of ITG modes [42] as well as ion-ion collisions.

Linear theory is not sufficient to explain reductions in turbulent transport since the saturation and non-linear regime of ITG modes involves effects such as the $\boldsymbol{E} \times \boldsymbol{B}$ convective non-linearity and selfgenerated poloidal flows; thus it must be demonstrated that there is also a reduction of turbulence in the non-linear regime. Furthermore, it must be proven that such a reduction is measurable by our diagnostics at the plasma edge. A nonlinear gyrokinetic particle-in-cell simulation model in toroidal geometry, which has been extended to include multiple ion species [42], is used to investigate the behaviour of turbulent ITG fluctuations and transport before and after impurity species are 


\section{J.A. Boedo et al.}
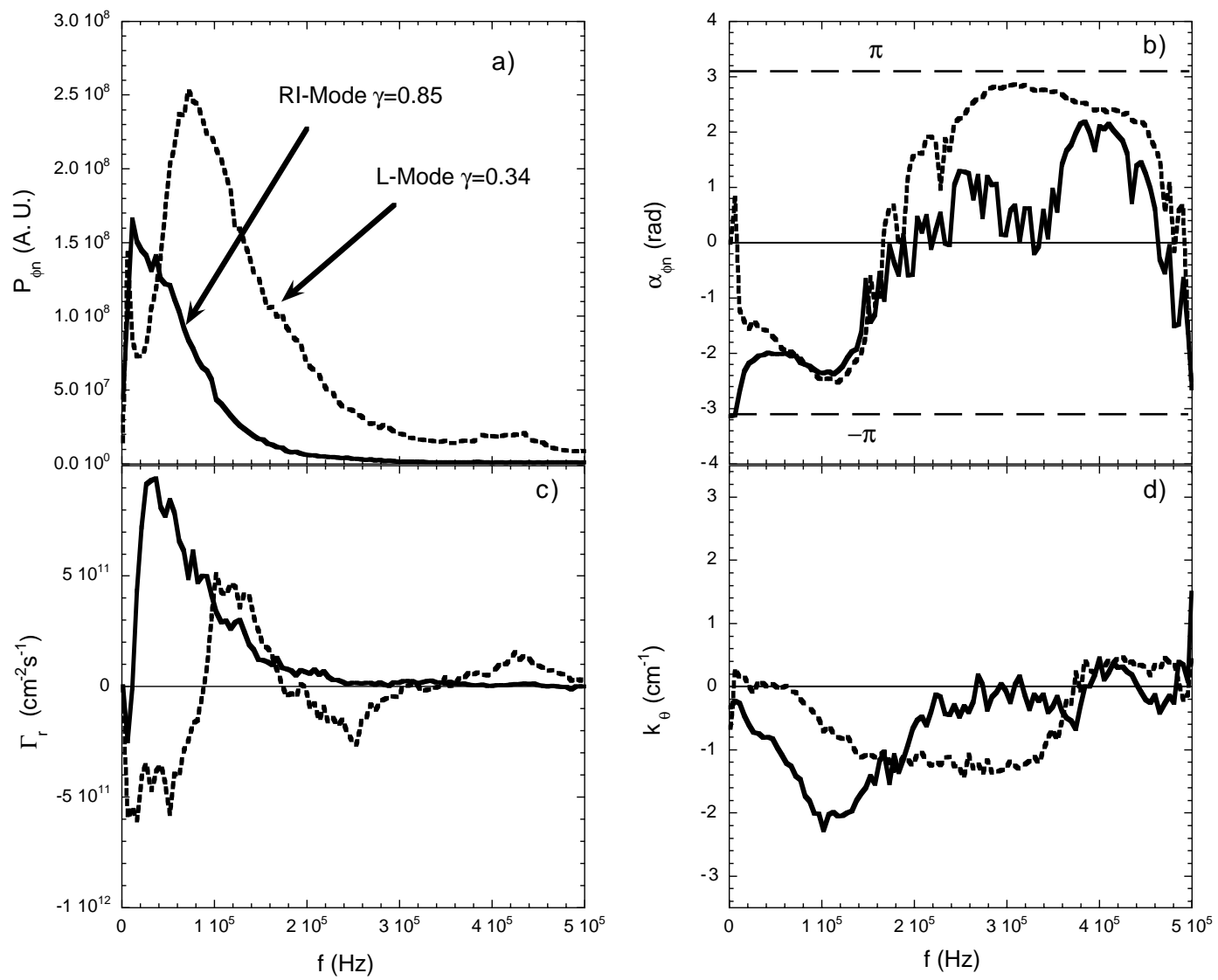

Figure 3. Frequency space plots of (a) cross-power spectrum, (b) cross-phase angle, (c) turbulent particle flux and (d) poloidal wavenumber $k_{\theta}$. Signals are strongly suppressed at frequencies higher than $100 \mathrm{kHz}$.

introduced into an L mode (and ITG unstable) TEXTOR plasma. The maximum number of toroidal mode numbers used was $n=40$ and the total number of particles was $10 \times 10^{6}$. The particle simulation algorithm was made highly efficient by parallelization using a domain decomposition method in the toroidal direction. The simulations were carried out using TEXTOR plasma parameters; aspect ratio $R / a=3.8$, minor radius $a \approx 200 \rho_{i}$ and poloidal wavenumber range $0.01<k_{\theta} \rho_{i} \leq 1$. Density, temperature, safety factor $q$, and impurity concentration profiles taken before and after neon injection from TEXTOR discharges are used as initial conditions.

Results from these simulations are shown in Fig. 4 indicating a strong suppression of the electrostatic fluctuations over the entire plasma volume and a reduction of the growth rate of the instabilities. Figure 4(a) shows the time evolution of the volume averaged electrostatic potential fluctuations in discharges with and without impurities. The electrostatic potential fluctuations are reduced by a factor of $\sim 10$ for a total neon concentration of $1 \%$ of the background density. The ion thermal flux drops by a factor of $4-5$. This result should be compared with that shown in Fig. 2, where a reduction in the relative fluctuation level of the potential by a factor of 2 is observed experimentally and with that of Fig. 1(d), where the RMS level of the poloidal electric field decreases by a factor of 2 . The turbulence suppression is observed across a large part of the plasma cross-section, mostly from $a / r=0.4-1.0$, as seen in Fig. 4(b), where the radial profile of the electrostatic potential is shown during the saturation phase of the turbulence. The extended effect over the crosssection is due to the fact that the higher charge states such as $\mathrm{Ne}^{8+}, \mathrm{Ne}^{9+}$ and $\mathrm{Ne}^{10+}$ have wide radial distributions, as shown in Fig. 5. The potential fluctuations in Fig. 4(b) are poloidally and time averaged over a correlation length/time $\left(t \omega_{c i}=(3-4) \times 10^{4}\right)$. 
Article: Turbulent transport and turbulence in RI mode
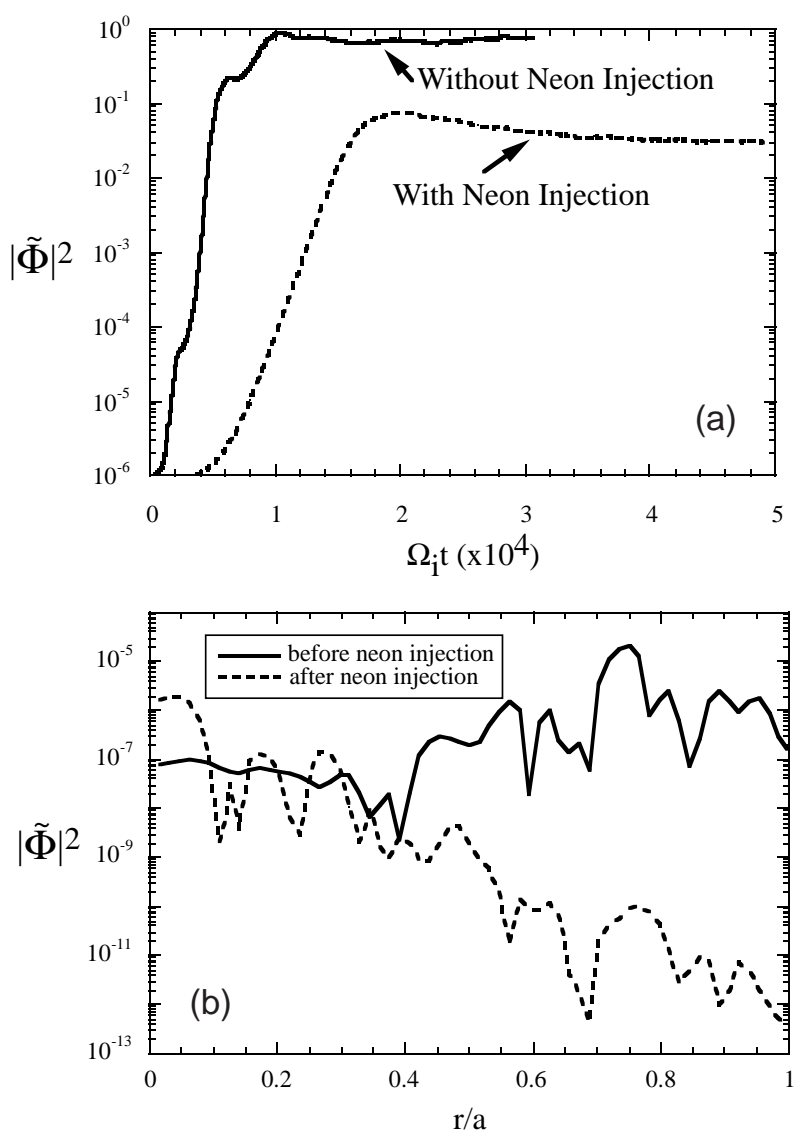

Figure 4. Evolution of potential fluctuations versus (a) time (time in multiples of $\omega_{c i}$ ) and (b) radius as calculated by the non-linear particle-in-cell gyrokinetic simulation. The system contains TEXTOR RI mode type plasmas as input with $1 \%$ neon content and without impurity seeding. The evolution of the system has been followed until saturation is reached.

It is important to note that the stabilization effects extend radially to the edge of the plasma, where the probe is located and our measurements taken. It is found that the fluctuation suppression is due to

(a) The combined effects of ion temperature profile modification due to the presence of the radiating impurity,

(b) Density peaking from reduced particle transport,

(c) Direct stabilization of the ITG modes via dilution.

Recent data [43] refute original claims of $\boldsymbol{E} \times \boldsymbol{B}$ shear [44] playing a dominant role in the core of TEXTOR during RI mode, however, since the ITG mode growth rate is reduced by impurity seeding effects (Eq. (1)), the existing $\boldsymbol{E} \times \boldsymbol{B}$ shear rate might

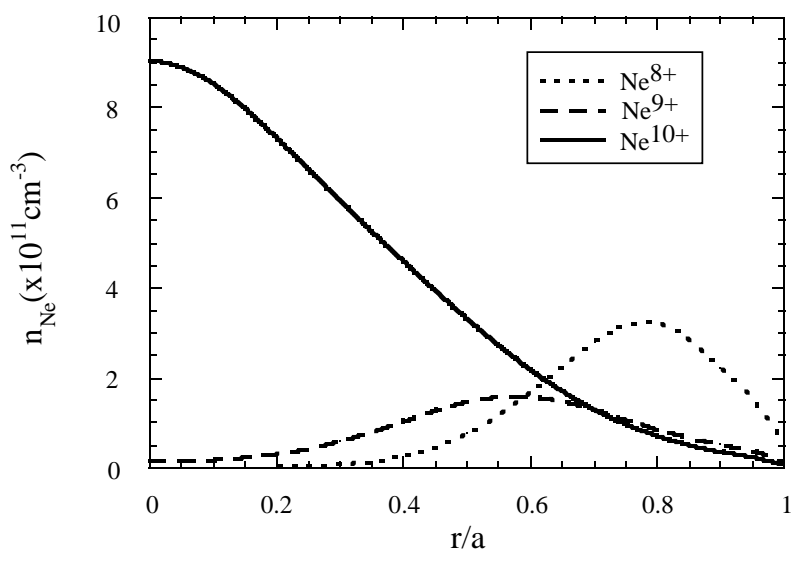

Figure 5. Radial profiles for the higher states of neon. The higher ionized states of neon, $\mathrm{Ne}^{8+}, \mathrm{Ne}^{9+}$ and $\mathrm{Ne}^{10+}$, have a wide radial distribution.

contribute to further stabilize the ITG modes. The signatures of $\boldsymbol{E} \times \boldsymbol{B}$ shear stabilization:

(i) Strong reduction (factors of 5) in the $E_{\theta r m s}$ levels,

(ii) Reduction in density fluctuation levels,

(iii) Finally, and most importantly, change in the phase angle between $E_{\theta r m s}$ and $n_{r m s}$ that causes a strong reduction of the radial particle flux,

are not observed in our data during RI mode discharges. Therefore, stabilization of turbulence by a shear layer in the edge or an internal shear layer that affects the edge by non-local effects $[45,23]$ due to long radial correlation lengths are not supported by known behaviour.

Another mechanism for the observed edge turbulence reduction could be the quenching of nonITG related turbulence related to the decrease in pressure, density and temperature gradients at the edge/SOL. Although we cannot completely disregard such a possibility, the non-linear simulations predict an ITG related reduction in the turbulence at the edge (and core) by factors of $\sim 5$ that are in agreement with observations. Furthermore, recent RI mode experiments in DIII-D [46] feature core and edge turbulence reductions that vary concomitantly with impurity type and amount, reinforcing the assumption that the mechanism at play is global.

\section{2. $\quad$ Edge and SOL profiles}

The reduction in radial particle transport and increased radiative losses result in a lower particle 


\section{J.A. Boedo et al.}
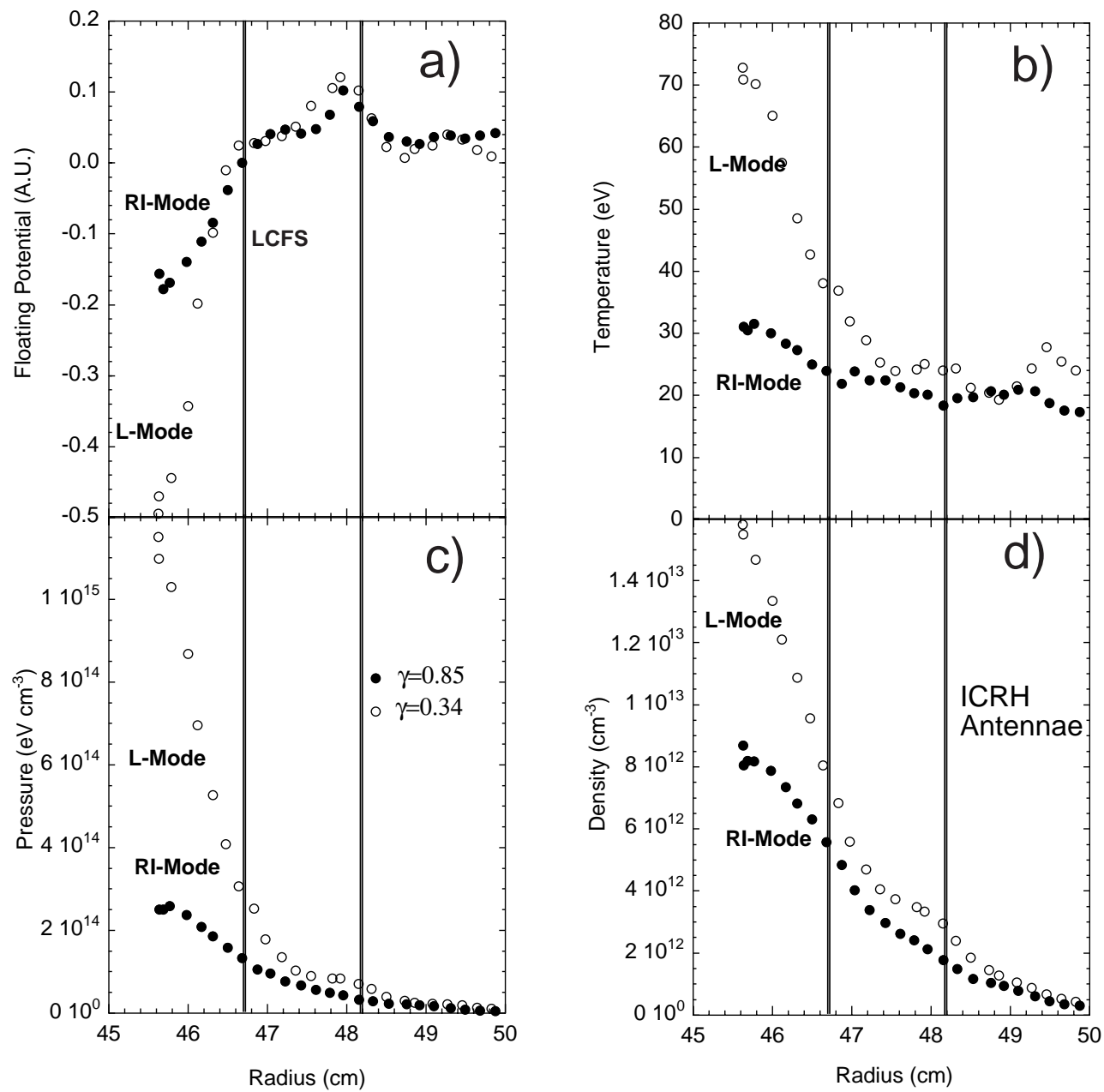

Figure 6. Edge radial profiles of (a) floating potential, (b) temperature, (c) electron pressure and $(\mathrm{d})$ density are shown for two discharges with L mode $(\gamma=0.34)$ and RI mode $(\gamma=0.85)$ confinement. The LCFS and the radial regions for fitting the profiles are indicated.

and energy inventory available for the edge and SOL, which determine the capabilities of the RI mode to ameliorate heat and particle loads to the plasma facing components and properly supply particles to the pumping apparatus. The edge and SOL $T_{e}$ and $n_{e}$ profiles (Figs 6(a),(d)) were measured with high radial resolution $(1.5 \mathrm{~mm})$ with the scanning probe in order to address the above issues.

The density profiles in the SOL (Fig. 6(d)) exhibit two distinct exponential decay lengths; the first between the LCFS and $r \sim 48 \mathrm{~cm}$ and the second (which is associated with other objects in the SOL such as the RF antennas and/or ponderomotive effects) between $r \sim 48 \mathrm{~cm}$ and the liner wall. Fits to the first decay length, herein dubbed local fits, and to the overall SOL profile, yield values of temperature $T_{e b}$, and density $n_{e b}$, at the LCFS and profile decay lengths, $\lambda_{n}$ and $\lambda_{n}^{\text {local }}$, which show similar behaviour with plasma parameters but can differ in absolute value. Typical decay lengths in the SOL are $0.6-1.5 \mathrm{~cm}$; thus the position of the LCFS, and of the profile resolution, must be known precisely to obtain accurate fits. The drop in the floating potential $V_{f}$ (Fig. 6(a)) allows an accurate determination of the position of the LCFS within $2 \mathrm{~mm}$, and it is found that the discharge to discharge variation of this position is as high as $10 \mathrm{~mm}$, or nearly $100 \%$ of the density decay length.

Both edge density and temperature (Fig. 7(a,b)) are reduced by factors of $2-3$ as the energy available at the edge diminishes due to radiation. The temperature profile (Fig. 6(b)) during the RI mode is very flat with a superimposed decay length $\lambda_{T} \sim$ $0.5 \mathrm{~cm}$ in the SOL which is fairly insensitive to the 
Article: Turbulent transport and turbulence in RI mode
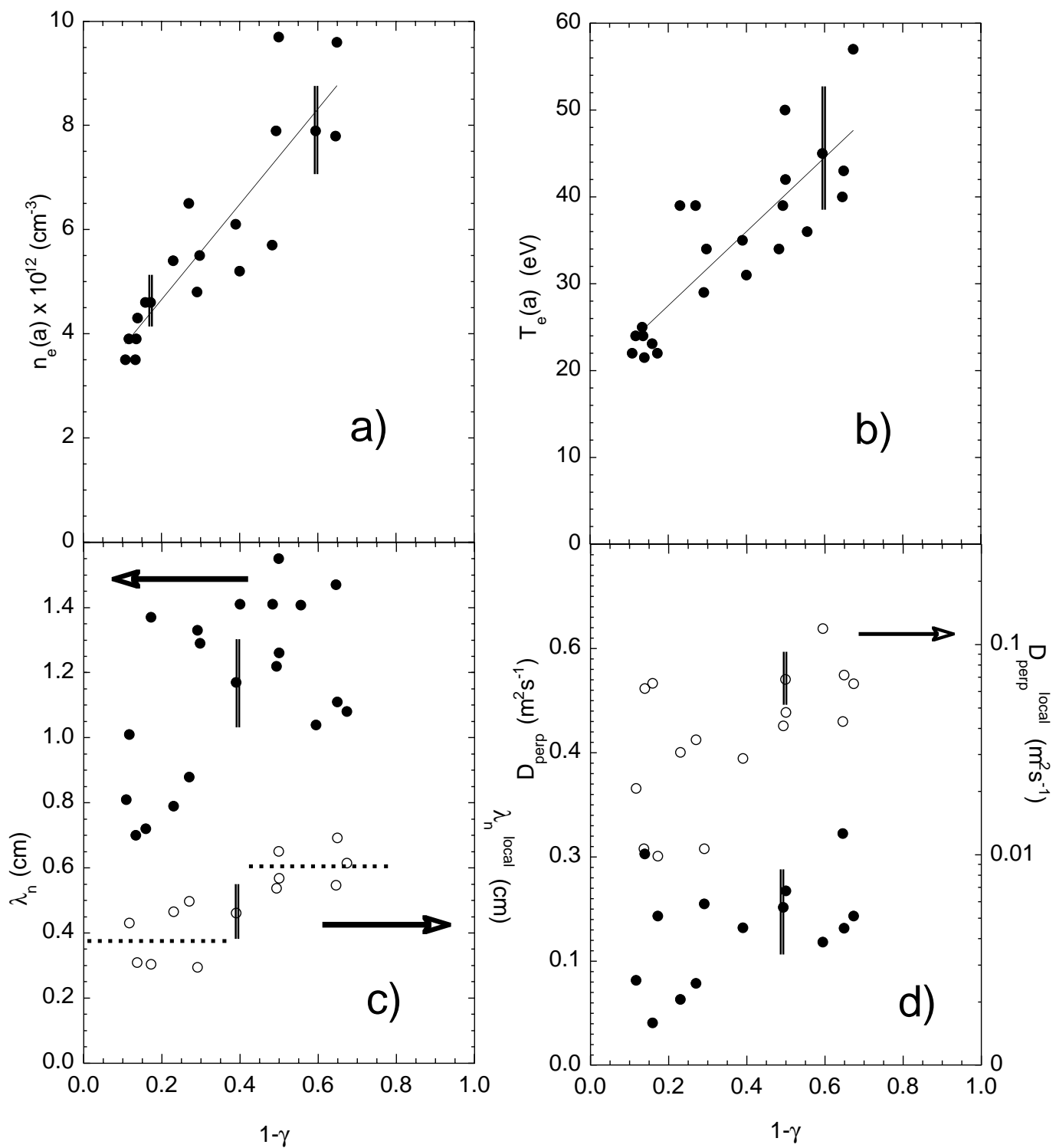

Figure 7. Scalings with radiated fraction of (a) electron density $n_{e b}$ and (b) electron temperature $T_{e b}$ measured at $r=a$, (c) local and global decay lengths and (d) perpendicular diffusion coefficients.

radiated fraction within the significant data scatter. The flatness of the profile indicates either that there is a significant power source, or sink, in the SOL or that the ratio of perpendicular to parallel thermal conductivity is higher than that in L mode.

Accurate edge parameters allow us to calculate the power flux at the LCFS, which can be written in terms of the sheath transmission factor $\delta$ (which is $\sim 8$ ), the boundary density $n_{b}$ and temperature $T_{e b}$, and the sound speed $c_{s}(a)$, as

$q_{\|}=\delta n_{b} c_{s}(a) T_{e b}$.
The reduction in edge density and temperature result in diminished power flux to the limiter (Fig. 8) by up to a factor of 7 ; the power flux features a linear decrease with radiation fraction.

The density decay lengths, extracted from the local and global exponential fits, are reduced by a factor of 1.5-2 as shown in Fig. 7(c), reflecting reduced radial particle fluxes. Although both decay lengths behave similarly with radiative fraction, there is a factor of 2-3 difference between them. We emphasize that the decay length dubbed 'local' is considered to be more accurate than the global one. The changes 


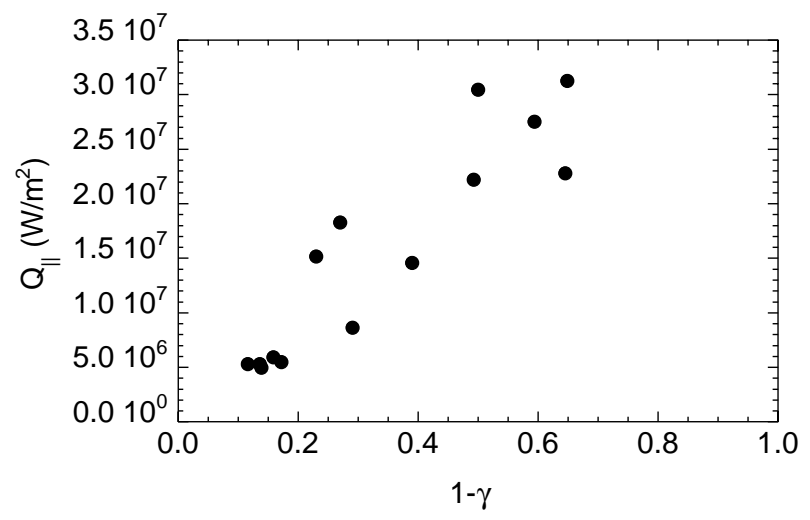

Figure 8. Heat flux convected to the limiter $\left(\mathrm{W} / \mathrm{m}^{2}\right)$ is reduced by a factor of 7 during $\mathrm{RI}$ mode conditions.

in radial transport can be quantified by calculating a perpendicular diffusion coefficient $D_{\perp}$ as a function of the density decay length $\lambda_{n}$, the sound speed $c_{s}$ and the parallel connection length $L_{\|}$,

$D_{\perp}=\frac{\lambda_{n}^{2} c_{s}}{2 L_{\|}}$.

We obtain values of $D_{\perp}$ and $D_{\perp}^{\text {local }}$ corresponding to the use of either $\lambda_{n}$ or $\lambda_{n}^{\text {local }}$ and shown in Fig. 7(d) for a connection length of $\sim 20 \mathrm{~m}$. The diffusion coefficient in the SOL is of the order of $0.4 \mathrm{~m}^{2} \mathrm{~s}^{-1}$, or about half the Bohm value, and decreases markedly with radiated power; this indicates a reduction in radial particle transport by factors of $2-3$, all of which is consistent with the decreased radial turbulent particle transport.

It is important to note that the reduction in heat flux is concomitant with a reduction in density and density decay length, which results in a lower particle flux to the pumping apparatus. The integrated flux (integrated over the SOL width) to the ALT-II limiter during the RI mode is $15 \%$ of the $\mathrm{L}$ mode flux. The reduction in density decay length from $\lambda_{n} \approx(1 / 2-1) \Delta_{L}$ to $\lambda_{n} \approx(1 / 4-1 / 2) \Delta_{L}$ (where $\Delta_{L}$ is the radial thickness of the limiter head) starves of particles the collection apparatus of the pump limiter [36], which is located behind the limiter surface. A divertor can handle this issue by positioning of the strike point at the pump.

\subsection{Particle and energy confinement}

The reduction in radial particle transport occurs across the LCFS, decreasing the particle losses from the main plasma and contributing to the increased particle confinement time that can be calculated [47]

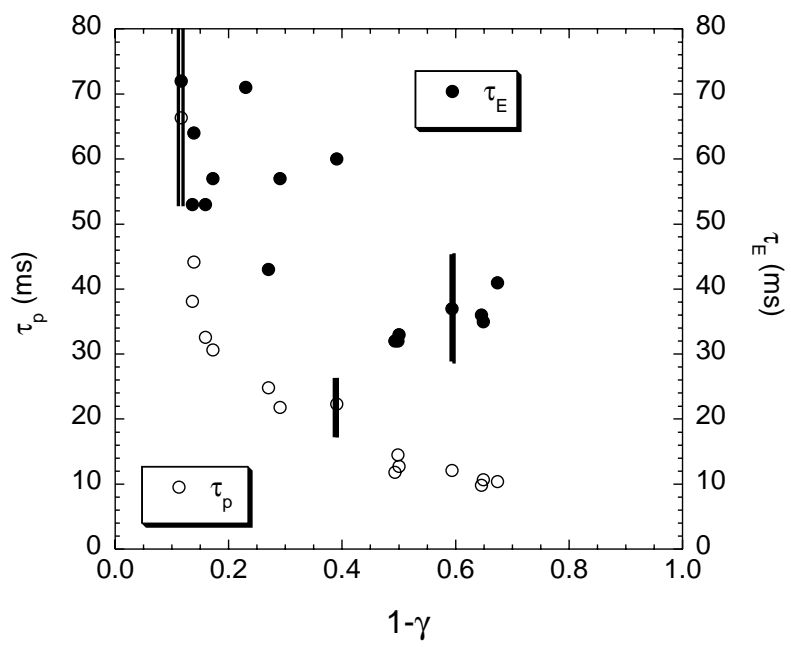

Figure 9. Particle $\tau_{p}$ and energy $\tau_{E}$ confinement times as a function of the radiated fraction. The confinement times increase by a factor of $\sim 3-5$ for $\gamma \geq 0.5$ as the discharges enter RI mode.

by relating the edge temperature $T_{e b}$, input power $P_{t o t}$, radiated power $P_{\text {rad }}$, total number of particles $N_{\text {tot }}$ and sheath transmission factor $\delta$ as

$\tau_{p}=\frac{N_{t o t} T_{e b} \delta}{\left(P_{t o t}-P_{r a d}\right)}$.

The assumptions behind this calculation are: the parallel heat transport is convective, $T_{e b}$ is poloidally homogeneous, no significant sources of particles or heat exist in the SOL and the particle and heat wetted areas are roughly similar. The experimentally determined values of $P_{t o t}, P_{r a d}$ and $N_{t o t}$ from the TEXTOR diagnostic systems, and $T_{e b}$ from the fast scanning probe, are used. The total radiated power is obtained from integration of the thermographic reconstruction with $2 \%$ accuracy. Photoionization by UV neon radiation is calculated to be negligible (less than $2 \%$ ) in the SOL. The ratio of $T_{e}$ and $T_{i}$ in the edge is about 1.3, and thus the sheath transmission factor is taken to be $\sim 8$. The calculated $\tau_{p}$ is shown in Fig. 9 and compared with the energy confinement time $\tau_{E}$. Two main features emerge from this figure:

(a) Discharges at values of $\gamma$ below $0.5-0.6$ feature a nearly constant $\tau_{p}$,

(b) for $\gamma$ above $0.5-0.6$ the increase in $\tau_{p}$ is steep with $\gamma$, by up to a factor of 4 .

These observations are consistent with $\mathrm{H}_{\alpha}$ measurements and with RI mode discharges recovering Neo-Alcator scaling. The turbulent particle flux convected power is $20 \%$ that of the radiated power in $\mathrm{L}$ mode conditions (37\% radiated power) and drops 
Table 1. Key edge and core parameters that define the ratio of the particle and energy confinement times

\begin{tabular}{lcccccc}
\hline & $\gamma$ & $T_{e}(a)$ & $T_{e 0}$ & $\alpha_{t}$ & $\alpha_{n}$ & $\tau_{E} / \tau_{p}$ \\
\hline L mode & 0.34 & 40 & 1400 & 2.5 & 0.75 & 4.1 \\
RI mode & 0.85 & 25 & 1350 & 2.26 & 1.16 & 1.7 \\
\hline
\end{tabular}

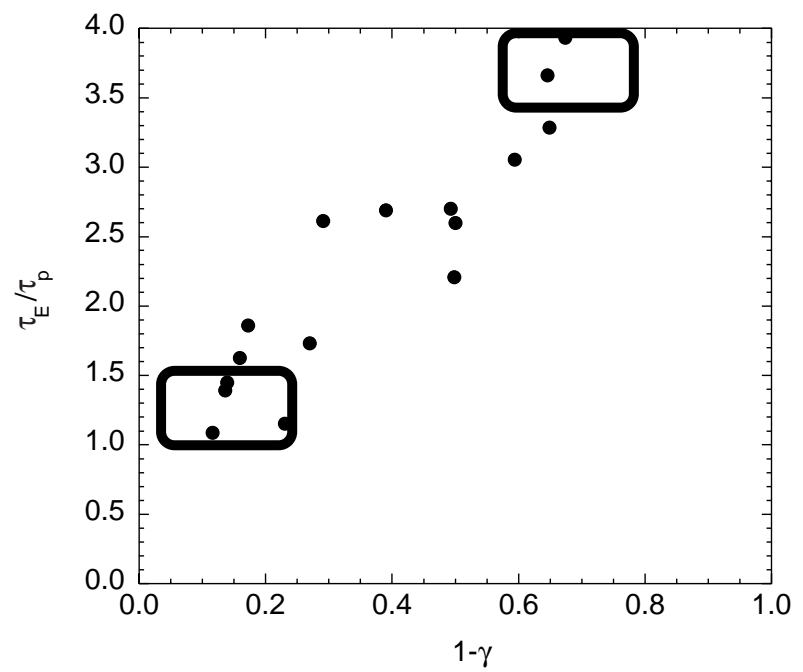

Figure 10. Ratio of energy and particle confinement times $\tau_{E} / \tau_{p}$ as a function of radiated fraction. The relationship found is linear. The calculated ratios from Eq. (8) are marked with boxes.

to $1 \%$ of the radiated power in RI mode conditions (95\% radiated power); thus the particle confinement does not play a critical direct role in the energy exhaust.

Not only $\tau_{p}$ but also $\tau_{E}$ shows a sharp increase with radiated fraction [48], showing a good correlation between the increase in energy and particle confinement and indicating that the mechanism responsible for the enhancement affects both the particle and the energy confinement. The $\tau_{E} / \tau_{p}$ ratio exhibits a tendency to increase with density in RI mode discharges, as shown in Fig. 10, meaning that $\tau_{p}$ rises faster with radiated power than $\tau_{E}$. It is of interest to understand, and to investigate, the possibility of controlling $\tau_{E} / \tau_{p}$, because the particle confinement affects the SOL critically (and particle and power exhaust) and a related quantity, $\rho^{*}=\tau_{p}^{*} / \tau_{E}$, has relevance for helium exhaust. The energy and particle confinement times are related by an expression [49] including the total energy content $E$, the total number of particles $N_{t o t}$, the input power $P_{t o t}$, radiated power $P_{\text {rad }}$, the boundary temperature in electronvolts, $T_{e b}$ and the sheath transmission factor $\delta$,

$$
\frac{\tau_{E}}{\tau_{p}}=\frac{E\left(P_{i n}-P_{\text {rad }}\right)}{\delta T_{b} N P_{i n}}=\frac{E(1-\gamma)}{\delta T_{b} N} .
$$

The energy content and total number of particles are obtained by integration of the density and temperature profiles, which are assumed parabolic with a shaping factor $\alpha$, and written in terms of the peak density $n_{e 0}$ and temperature $T_{e 0}$ for a plasma of major radius $R_{0}$ and minor radius $a$. The suffixes $e$ and $i$ denote ions and electrons, respectively. We write for the electrons,

$$
\begin{aligned}
& n_{e}(r)=n_{e 0}\left[1-\left(r-R_{0}\right)^{2} / a^{2}\right]^{\alpha_{n}} \\
& T_{e}(r)=T_{e 0}\left[1-\left(r-R_{0}\right)^{2} / a^{2}\right]^{\alpha_{T}} .
\end{aligned}
$$

Fits to the measured density and temperature profiles equation (7) show increased peaking during the well developed RI mode, as shown in Table 1. We can include the profile shapes (Eq. (7)) in Eq. (6) to obtain Eq. (8), which can be simplified further (Eq. (8)) by setting $T_{i 0}=T_{e 0}$ and $\alpha_{i}=\alpha_{e}$, as expected from the core conditions,

$$
\begin{aligned}
\frac{\tau_{E}}{\tau_{p}}= & \frac{3}{2}\left(\frac{1-\gamma}{\delta}\right)\left(\frac{1+\alpha_{n}}{T_{b}}\right) \\
& \times\left(\frac{T_{i 0}}{1+\alpha_{n}+\alpha_{i}}+\frac{T_{e 0}}{1+\alpha_{n}+\alpha_{e}}\right) \\
\frac{\tau_{E}}{\tau_{p}}= & 3\left(\frac{1}{\delta}\right)(1-\gamma)\left(\frac{T_{i 0}}{T_{e b}}\right)\left(\frac{1+\alpha_{n}}{1+\alpha_{n}+\alpha_{e}}\right) \\
= & 3\left(\frac{(1-\gamma)}{T_{e b}}\right)\left(\frac{T_{i 0}}{\delta}\right)\left(\frac{1+\alpha_{n}}{1+\alpha_{n}+\alpha_{e}}\right) .
\end{aligned}
$$

When data from profiles (Table 1) are entered into Eq. (9), it is found that the confinement time ratio changes by a factor of 2.2 between L mode $(\gamma=37 \%)$ and RI mode $(\gamma=95 \%)$, in agreement (Fig. 10) with the ratio of measured confinement times. The observed change in the ratio $\tau_{E} / \tau_{p}$ is mildly dependent $(10 \%)$ on the profile shaping (Table 1) and strongly dependent on the ratio $(1-\gamma) / T_{e b}$, i.e. the ratio of the radiated fraction to the boundary temperature, mainly due to the fact that there is a larger reduction in $1-\gamma$ (factor of 3-5) than in $T_{e b}$ (factor of 2) with increased radiated power. However, the decrease of $\tau_{E} / \tau_{p}$ with $\gamma$ can be counteracted by further reducing $T_{e b}$ by either increasing the density [50] or by using higher- $Z$ impurities or a mix of impurities that determine a lower $T_{e b}$. The $\tau_{E} / \tau_{p}$ ratio can be changed by a factor of 30 by tuning the edge temperature from 12 to $400 \mathrm{eV}$. 


\section{J.A. Boedo et al.}

\section{Conclusions}

This article presents the first measurements of turbulence and turbulent transport in the edge of impurity seeded discharges exhibiting RI mode confinement. The turbulent radial transport is reduced due to quenching of the levels of density and potential fluctuations, particularly at high frequencies. No evidence for velocity shear stabilization is found in the outer edge of the plasma. The measurements have been compared with results from a fully nonlinear gyrokinetic simulation of ITG modes in the same discharges. The simulation predicts a reduction of turbulence across the full cross-section, including the edge; therefore we propose stabilization of ITG modes as a mechanism to explain the reduction in turbulence in RI mode discharges. These results have wide application in toroidal confinement devices and ongoing work will strive to separate the different mechanisms at play, with the aim to improve the performance of fusion devices.

The reduction of turbulence causes an increase in both energy and particle confinement which produce a marked reduction in temperature and density in the plasma edge during the RI mode and result in a factor of nearly 10 reduction in the power and particle load to the plasma facing components. The stabilization is triggered at $\gamma \approx 0.6$. We find a linear relation for the $\tau_{E} / \tau_{p}$ ratio versus $\gamma$, which indicates a faster rise for particle than energy confinement. A 1-D calculation which reproduces the experimental ratio of $\tau_{E} / \tau_{p}$, and its scaling as a function of the radiated power fraction, indicates that the changes in the ratio $\tau_{E} / \tau_{p}$ are strongly dependent on the relation between the edge temperature and the radiated power.

\section{Acknowledgements}

This work has been supported by USDOE Grant No. DE-FG03-85 ER 51069. The authors want to express their gratitude to K. Burrell, T. Evans, G. Tynan and M. Schaffer for many useful interactions and to L. Russo for unwavering support.

\section{References}

[1] ASDEX Team, Nucl. Fusion 29 (1989) 1959.

[2] Taylor, R.J., et al., Phys. Rev. Lett. 63 (1989) 2365.

[3] Biglari, H., et al., Phys. Fluids B 2 (1990) 1.

[4] Ritz, C.P., et al., Phys. Rev. Lett. 65 (1990) 2543.
[5] Ongena, J., et al., in Controlled Fusion and Plasma Physics (Proc. 20th Eur. Conf. Lisbon, 1993), Vol. 17C, Part I, European Physical Society, Geneva (1993) 127.

[6] Messiaen, A., et al., Phys. Rev. Lett. 77 (1996) 2487.

[7] Samm, U., et al., in Controlled Fusion and Plasma Physics (Proc. 18th Eur. Conf. Berlin 1991), Vol. 15C, Part III, European Physical Society, Geneva (1991) 157.

[8] Samm, U., et al., Plasma Phys. Control. Fusion 35 (1993) 167.

[9] Ongena, J., et al., Plasma Phys. Control. Fusion 41 Suppl. 3A (1999) 379.

[10] Taylor, T.S., Plasma Phys. Control. Fusion 39 Suppl. 12B (1997) 47.

[11] Burrell, K.H., et al., Plasma Phys. Control. Fusion 34 (1992) 1859.

[12] Burrell, K.H., et al., Phys. Plasmas 1 (1994) 1536.

[13] Synakowski, E.J., et al., Phys. Plasmas 4 (1997) 1736 .

[14] Synakowski, E., et al., Phys. Rev. Lett. 78 (1997) 2972.

[15] Moyer, R., et al., Phys. Plasmas 2 (1995) 2397.

[16] Tynan, G.R., et al., Phys. Plasmas 1 (1994) 3301.

[17] Weynants, R.R., et al., paper presented at IAEA Tech. Comm. Mtg, Kloster Seeon, 1991.

[18] Weynants, R.R., et al., Nucl. Fusion 32 (1992) 837.

[19] Cornelis, J., et al., Nucl. Fusion 34 (1994) 171.

[20] Weynants, R.R., et al., in Plasma Physics and Controlled Nuclear Fusion Research 1990 (Proc. 13th Int. Conf. Washington, DC, 1990), Vol. 1, IAEA, Vienna (1991) 473.

[21] Jachmich, S., et al., Plasma Phys. Control. Fusion 40 (1997) 1105.

[22] Tynan, G.R., et al., J. Nucl. Mater. 196-198 (1992) 770 .

[23] Gray, D.S., et al., Nucl. Fusion 38 (1998) 1585.

[24] Massey, R.S., et al., Fusion Technol. 8 (1985) 1571.

[25] Najmabadi, F., et al., Fusion Eng. Des. 23 (1993) 69.

[26] Conn, R.W., et al., in Plasma Physics and Controlled Nuclear Fusion Research 1988 (Proc. 12th Int. Conf. Nice 1988), Vol. 3, IAEA, Vienna (1989) 315.

[27] Carraro, L., et al., in Controlled Fusion and Plasma Physics (Proc. 25th Eur. Conf. Prague, 1998), Vol. 22C, European Physical Society, Geneva (1998) 790.

[28] Lazarus, E.A., et al., J. Nucl. Mater. 121 (1984) 61.

[29] Lazarus, E.A., et al., Nucl. Fusion 25 (1985) 135.

[30] Ongena, J., et al., Comments Plasma Phys. Control. Fusion 18 (1998) 221.

[31] Paccagnella, R., Romanelli, F., Nucl. Fusion 30 (1990) 545.

[32] Dominguez, R.R., Nucl. Fusion 31 (1991) 2063. 
[33] Fröjdh, M., Liljeström, M., Nordman, H., Nucl. Fusion 32 (1992) 419.

[34] Dominguez, R.R., Nucl. Fusion 31 (1991) 2063.

[35] Fröjdh, M., Liljeström, M., Nordman, H., Nucl. Fusion 32 (1992) 419.

[36] Goebel, D.M., et al., J. Nucl. Mater. 162-164 (1989) 115.

[37] Samm, U., et al., Plasma Phys. Control. Fusion 35 (1993) B167.

[38] Dippel, K.H., J. Nucl. Mater. 145-147 (1987) 3.

[39] Boedo, J., et al., Rev. Sci. Instrum. 69 (1998) 2663.

[40] Bickerton, R.J., Nucl. Fusion 13 (1973) 290.

[41] Powers, E.J., Nucl. Fusion 14 (1974) 749.

[42] Sydora, R.D., Decyk, V.K., Dawson, J.M., Plasma Phys. Control. Fusion 38 (1996) A281.

[43] Jaspers, R., et al., in Controlled Fusion and Plasma Physics (Proc. 25th Eur. Conf. Prague, 1998), Vol. 22C, European Physical Society, Geneva (1998) 552.
[44] Unterberg, B., et al., Plasma Phys. Control. Fusion 39 (1997) B189.

[45] Burrell, K., General Atomics, San Diego, CA, personal communication, 1998.

[46] McKee, G., General Atomics, San Diego, CA, personal communication, 1999.

[47] Stangeby, P.C., McCracken, G.M., Nucl. Fusion 30 (1990) 1264.

[48] Vandenplas, P.E., Messiaen, A.M., Ongena, J.P., Samm, U., Unterberg, B., J. Plasma Phys. 59 (1998) 587.

[49] Gray, D.S., Boedo, J.A., Conn, R., Nucl. Fusion 37 (1997) 1437.

[50] Messiaen, A., et al., Phys. Plasmas 4 (1997) 1690.

(Manuscript received 30 August 1998

Final manuscript accepted 8 October 1999)

E-mail address of J. Boedo: jboedo@ferp.ucsd.edu

Subject classification: D2, Te; F2, Te; I1, Te 